\title{
Symposium review: Microbial endocrinology-Why the integration of microbes, epithelial cells, and neurochemical signals in the digestive tract matters to ruminant health ${ }^{1}$
}

\author{
Mark Lyte, ${ }^{* 2}$ Daniel N. Villageliú, ${ }^{*}$ Brian A. Crooker, $†$ and David R. Brown $\ddagger$ \\ *Department of Veterinary Microbiology and Preventive Medicine, lowa State University, Ames 50011 \\ †Department of Animal Science, and \\ fDepartment of Veterinary and Biomedical Sciences, University of Minnesota, St. Paul 55108
}

\begin{abstract}
The union of microbiology and neurobiology, which has been termed microbial endocrinology, is defined as the study of the ability of microorganisms to produce and respond to neurochemicals that originate either within the microorganisms themselves or within the host they inhabit. It serves as the basis for an evolutionarily derived method of communication between a host and its microbiota. Mechanisms elucidated by microbial endocrinology give new insight into the ways the microbiota can affect host stress, metabolic efficiency, resistance to disease, and other factors that may prove relevant to the dairy industry.
\end{abstract}

Key words: epithelium, microbiology, infection, microbiota-gut-brain axis

\section{CONNECTION BETWEEN THE GUT MICROBIAL COMMUNITY AND STRESS}

Animals experience a variety of stressors that occur when they transition between physiological conditions, when they are exposed to changing environments, and when these and other stressors interact. Environmental stress, particularly heat stress, has long been established as influencing dairy animal performance in measures including growth, reproduction, and lactation (Collier et al., 2006). Heat stress during the transition to lactation can be especially detrimental to dairy cattle performance (Baumgard and Rhoads, 2012, 2013). Acclimation to stress in ruminants includes numerous endocrine changes, such as reduced aldosterone secretion, reduced glucocorticoid secretion, increased epinephrine,

Received July 28, 2017.

Accepted February 7, 2018.

${ }^{1}$ Presented as part of the Growth and Development Symposium: Microbial Endocrinology in Ruminant Growth and Development at the ADSA Annual Meeting, Pittsburgh, Pennsylvania, June 2017.

${ }^{2}$ Corresponding author: mlyte@iastate.edu and increased progesterone secretion by the adrenal glands; decreased thyroxine secretion by the thyroid gland; increased leptin secretion by adipose tissue; decreased somatotropin and increased prolactin secretion by the anterior pituitary; and decreased estrone sulfate secretion by the placenta (Bernabucci et al., 2010). Although extensive studies of rumen microbial ecology and the effect of dietary alterations on microbial communities, nutrient supply, and animal performance have been conducted, investigations into the effects of animal stressors on the microbiome of ruminants are in their infancy (Malmuthuge and Guan, 2017). Initial work has identified alterations in rumen microbiota when heifers were heat stressed (Uyeno et al., 2010), when cows were subjected to intravenous challenges with LPS (Jing et al., 2014), and when cows transition to lactation, especially when the rumen becomes acidotic (Wetzels et al., 2016). Given that links between stress, the neuroendocrine system, and the microbiota are well established, even if not completely understood in other mammals (Furness et al., 2013; Furness, 2016; Schroeder and Backhed, 2016), it is likely that similar relationships will be identified in ruminants.

Stress can act through the central nervous system and effect changes to the microbiota through the sympathoadrenal and hypothalamic-pituitary-adrenal axes (Lyte, 2016b). These neurochemical axes release chemicals that can act on and change the microbiota. Changes in the host's microbiota may then feedback onto the host through the gut-brain axis to effect further changes. Simply stated, the microbiota and host are intimately linked in a bidirectional way (Figure 1). A physiological model drawn from our understanding of the interactions of microbes with the endocrine and enteric nervous systems has been developed and is termed the microbiota-gut-brain axis. Food ingested by the host can be metabolized by the microbiota in ways that generate specialized metabolites that have the potential to modulate host health (Sharon et al., 2014). Neurochemical metabolites can be taken up into 


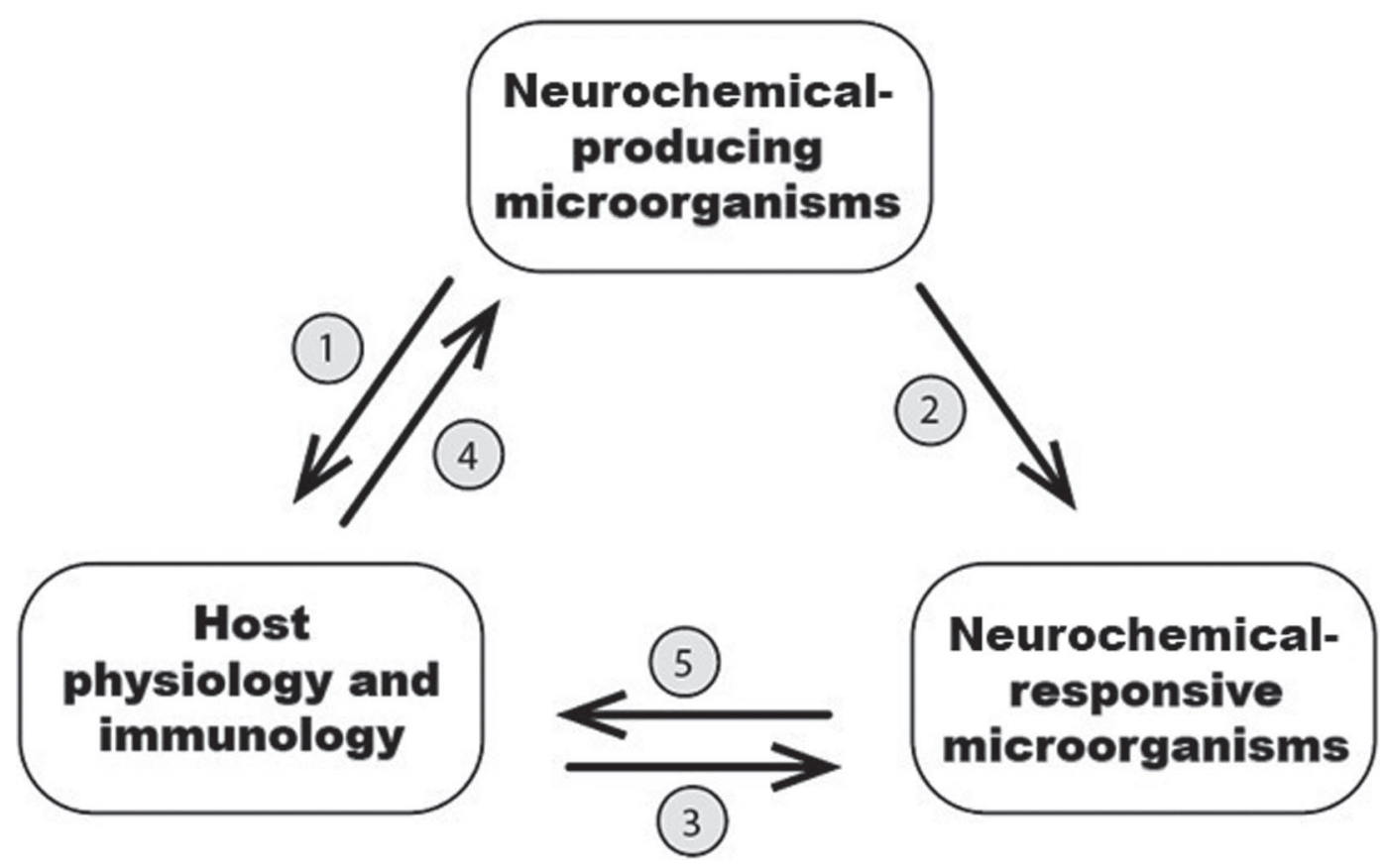

Figure 1. Bidirectional nature of microbial endocrinology in which neurochemicals produced by the host and microbiota can influence each other. Neurochemicals produced by the microbiota can influence the host (1) as well as responsive microorganisms within the overall microbial community in the alimentary tract (2). The host response to neurochemicals may include physiological changes (intestinal motility, water reabsorption, secretory), behavioral changes (appetite, mood), or immunological states (i.e., inflammation). Similarly, the microbiota can respond to neurochemicals secreted from either the host (such as during periods of stress; 3) or other microorganisms (2) through altered physiology (i.e., infection, secretion, or environmental tolerance). Physiological changes in the host may feedback onto the neurochemical producers of the microbiota (4). Neurochemical responsive members of the microbiota also have the capacity to generate a response that affects the host (5). Together these pathways have the capacity to lead to reciprocal responses, as indicated by the paired arrows, and are part of the more generalized microbiota-gut-brain axis (Lyte, 2014).

the circulation or interact with the host locally at the level of the enteric nervous system. Some signals may be transduced to the brain, where they can influence cognition and behavior, whereas other signals may result in changes in the enteric system, which feedback on the microbiota in a bidirectional way.

Among many potential signals that can pass between a host and the microbiota, the catecholamine molecule norepinephrine (NE) is considered to be of particular importance. For the purposes of this limited review, NE will serve as a prototypical example for the manner in which bacterial-neuroendocrine interactions function within the larger context of microbial endocrinology. Norepinephrine normally functions as a neurotransmitter in the digestive tract, but can act as a hormone as well when it is released from the adrenal medulla in response to a stressful stimulus and carried through the blood to the ruminal and intestinal epithelia. Norepinephrine is among several chemical mediators that may play an important role in modulating host-bacteria interactions (Hughes and Sperandio, 2008). Although many investigations of $\mathrm{NE}$ actions on the intestinal mucosa have been carried out, the majority of these have used rodent, rabbit, or guinea pig models and only a relatively small number have been conducted with livestock species, and those have been performed mainly on the porcine intestine. As few analogous studies exist in cattle, we reviewed host-bacteria interactions at the intestinal mucosa surfaces and NE-host-bacteria interactions, citing relevant studies in cattle when available.

\section{INTERDOMAIN COMMUNICATION IN THE INTESTINAL MUCOSA}

Through the production and release of chemical signals or by physical or structural interactions, the various members of the ruminal and intestinal microbial communities are capable of communicating with each other across taxonomic boundaries, including interdomain signaling between prokaryotes (bacteria) and eukaryotes (e.g., fungi). This microbial signaling extends to cells of the host as well, which is the subject of the emerging field of microbial endocrinology. In the rumen, bacteria have been observed to make direct contact with the stratum corneum of the stratified, squamous ruminal epithelium as well as rumen papillae (McCowan et al., 1978; Mueller et al., 1984). The surface of the stratum corneum is colonized by 
microbes in the rumen but, under normal conditions, these microbes do not penetrate this layer of dead cells and reach the stratum granulosum (Graham and Simmons, 2005; Steele et al., 2016). The glandular ruminal epithelium has no endocrine or resident immune cells and is sparsely innervated, at least in cattle (Kitamura et al., 1987; Kitamura et al., 1993; Teixeira et al., 1998).

In the intestinal tract, the simple columnar epithelium of the intestinal mucosa with its crypt-villus/ surface cell arrangement is the initial point of bacterial contact with the host. Goblet cells and crypt cells in the mucosa secrete mucin and water, respectively, to form inner and outer blankets of mucus harboring distinct populations of commensal microbes and ultimately protecting the mucosal surface from pathogen colonization in monogastrics (Atuma et al., 2001) and ruminants (Steele et al., 2016). The intestinal mucosa in particular has been a focus for investigations of interdomain crosstalk between bacteria and their mammalian hosts. It contains the largest number of endocrine cells in the body, which are diffusely distributed along its length, a substantial population of resident immune cells that provide innate and adaptive immune defense, and an intramural nervous system capable of supporting preprogrammed behavior that can be modulated by the central and autonomic nervous systems. Cell to cell communication occurs among different host cell types in or near the mucosa as well as with bacteria and other microorganisms. Additional knowledge of the role of the mucus in ruminants as a barrier and how it interacts with epimural microorganisms is needed (Bradford et al., 2015, 2016; Steele et al., 2016).

\section{NOREPINEPHRINE AFFECTS THE GUT MICROBIAL COMMUNITY VIA INTESTINAL MUCOSA}

Norepinephrine can act on adrenergic receptors located on submucosal nerves or the basolateral membranes of various cell types in the intestinal mucosa to influence bacterial interactions with the mucosal surface (Figure 2). First, in intestinal mucosa explants from swine, NE affects active transepithelial ion and fluid transport as measured by changes in short-circuit current. In the small intestine, it acts through alpha-2 adrenergic receptors on submucosal enteric nerves to decrease changes in short-circuit current, an effect attributed to increased chloride absorption. Its effects on the colonic mucosa are quite different, as it acts directly on colonic epithelial cells to increase active chloride secretion; this latter effect is mediated by alpha- 1 adrenergic receptors (Brown and O'Grady, 1997). These effects of ion transport, with the accompanying movement of water through aquaporin channels, could influence the viscosity and thickness of the mucus layers covering the mucosa in these 2 intestinal regions and, by extension, affect the commensal microbial populations residing in the inner and outer mucus layers.

Second, NE appears to promote the vectorial movement of secretory immunoglobulin A (SIgA) through epithelial cells onto the mucosal surface and into the overlying mucus layers. At these locations, SIgA has important roles in retaining and clearing pathogenic bacteria to promote an anti-inflammatory environment, which is critical for preserving intestinal barrier function, as well as shaping and maintaining homeostasis in the commensal microbial community (Sutherland et al., 2016). In mucosal explants from swine small intestine and colon, at low micromolar concentrations, NE appears to accelerate the rate at which the polymeric IgA receptor ferries dimeric IgA from plasma B cells in the lamina propria to the mucosal surface. This effect appears to be mediated by alpha- 1 adrenergic receptors on mucosal epithelial cells (Schmidt et al., 2007). It is not clear if this process occurs in vivo, as an earlier investigation suggests that $\mathrm{NE}$, acting through a nervous mechanism, decreases SIgA output from the isolated, perfused porcine ileum (Schmidt et al., 1999).

Third, NE reversibly promotes adherence of pathogenic (Escherichia coli O157:H7) and some strains of nonpathogenic Escherichia coli to the mucosal surface of the cecal and colonic mucosa explants from swine (Green et al., 2004; Chen et al., 2006). It appears to enhance rapid, nonintimate association of these bacteria to the colonic mucosal surface by acting on epithelial alpha-2 adrenergic receptors with a 50\% effective concentration of approximately $1 \mu M$. This adrenergic receptor subtype is coupled through $\mathrm{G}$ proteins to a decrease in cyclic AMP levels, with a corresponding decrease in protein kinase A activity. Indirectly acting sympathomimetic drugs, such as tyramine and the protein kinase A inhibitor Rp-cAMPS, mimic this NE action. The underlying mechanism has not been discovered, although it is hypothesized that NE and its downstream intracellular effectors unveil a bacterial adherence factor, such as extracellular matrix proteins or mannose-binding proteins, on the luminal aspect of mucosal epithelial cells (Green et al., 2004).

In summary, NE may act through the intestinal mucosa at low $(1-10 \mu M)$ concentrations to promote mucosal interactions with bacteria in the gut lumen, through at least 3 mechanisms that are mediated by conventional $\alpha$-adrenergic receptors. First, hydrationassociated changes in the thickness of mucus layers covering the mucosa; second, increased SIgA delivery to the mucosal surface through actions on polymeric IgA receptor and luminal ionic and fluid movements; and third, promotion of early bacterial adherence to the mucosal surface. 


\section{DIRECT ACTIONS OF NOREPINEPHRINE ON INTESTINAL BACTERIAL CELLS}

Norepinephrine present in the intestinal lumen does not appear to arise solely from the host. Evidence that microbes in vivo can synthesize various biogenic amines, such as NE, has been shown in rodents (Asano et al., 2012). Given that the in vitro production of biogenic amines by bacterial genera used in food production (and which are also found in the gastrointestinal tract of many mammalian species) has been reported for decades (Silla Santos, 1996; Pessione and Cirrincione, 2016), it is likely that the microbiota of other species can also produce physiologically relevant levels of biogenic amines that may influence host physiology. At high $(>50 \mu M)$ concentrations, NE can act directly on bacteria to influence their chemotaxis toward the mu- cosal surface as well as enhance their growth and virulence. Lyte and Ernst (1992) were the first investigators to report the direct effects of NE on the growth and virulence characteristics of bacteria (E. coli) in vitro. In their original studies as well as in subsequent investigations of these phenomena in E. coli and many other bacterial species, NE produced its effects on growth and virulence at concentrations $>50 \mu M$, which are considerably higher than the concentrations of NE effective in altering intestinal mucosal function. However, extended incubation $(18 \mathrm{~h})$ of Pseudomonas aeruginosa with NE or epinephrine at concentrations as low as 5 $\mu M$ has been reported to increase bacterial numbers (Freestone et al., 2012). Moreover, lower concentrations of epinephrine $(\geq 5 \mu M)$ can affect intracellular events in E. coli, such as phosphorylation or gene transcription (Clarke et al., 2006). Some studies have reported
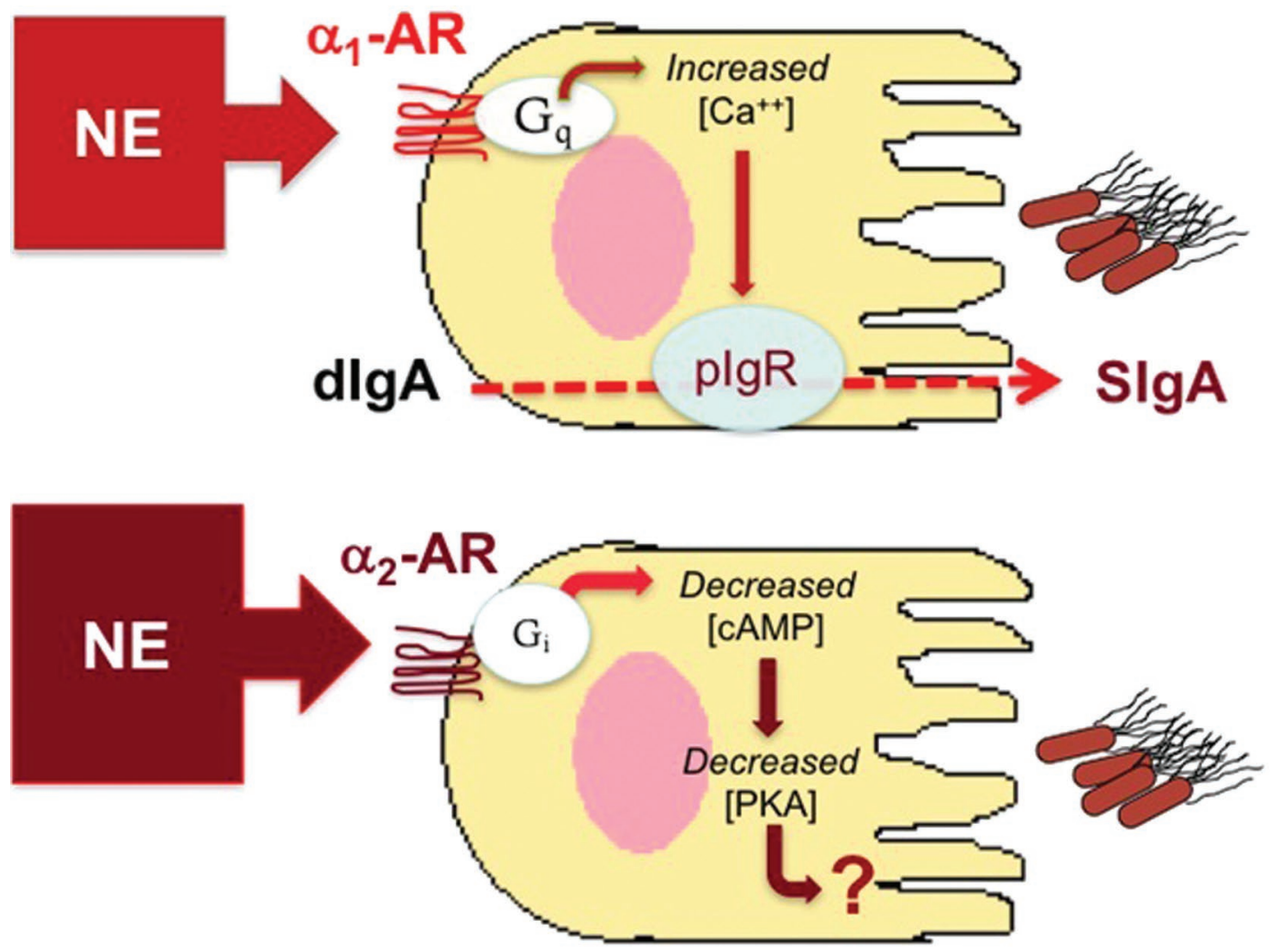

Figure 2. Effects of norepinephrine (NE) acting on epithelial cells at low micromolar concentrations in altering host-microbe interactions at the intestinal mucosal surface. In the top image, in explants of intestinal mucosa (small intestine and colon from swine), NE acts on basolateral alpha-1 adrenergic receptors $\left(\alpha_{1}-\mathrm{AR}\right)$ to increase transepithelial transport of dimeric immunoglobulin A (dIgA) to the mucosal surface as secretory IgA (SIgA). This calcium-mediated action appears to be due to an enhanced shuttling of the polymeric IgA receptor (pIgR) from the epithelial cell basolateral membrane to the apical membrane. In the bottom image, NE promotes nonintimate adherence of Escherichia coli to the mucosal surface of the cecal and colonic mucosa explants from swine by acting on basolateral alpha- 2 adrenergic membrane receptors $\left(\alpha_{2}-\mathrm{AR}\right)$ coupled to decreases in intracellular cyclic AMP levels and protein kinase A (PKA) activity. There exist multiple alpha subunit proteins that form part of larger, heterotrimeric GTP-binding proteins (or G proteins). These proteins link receptor stimulation to the activation of various signaling pathways in epithelial cells. $G_{i}$ is termed an inhibitory alpha subunit of a $G$ protein because it inhibits the formation of the second messenger, cyclic AMP. $\mathrm{G}_{\mathrm{q}}$ is a completely different alpha subunit of a $\mathrm{G}$ protein that was isolated by Mono Q anion-exchange chromatography in the early 1990s, hence the subscripted q. Color version available online. 
that specific adrenergic antagonists, particularly $\alpha$-adrenergic antagonists such as phentolamine, can inhibit the direct actions of NE on bacteria, but the antagonist concentrations required to do so are very high (Clarke et al., 2006). This suggests that conventional adrenergic receptors are not expressed on bacteria and do not mediate NE actions.

How does NE influence bacterial function, if not through specific adrenergic receptors? First, bacterial catecholates such as enterobactin and salmochelin maintain bacterial growth by binding to ferric iron and delivering it to bacteria under iron-restricted conditions (Ellermann and Arthur, 2017). Similar to these microbially-generated substances, NE contains a catecholate moiety, and it too possesses the characteristics of a siderophore. This mechanism appears to explain some of its effects on bacterial growth (Freestone et al., 2012).

Second, in addition to functioning as siderophores, catecholamines can facilitate the liberation of iron from the iron-binding proteins lactoferrin and transferrin. Catecholamines form direct complexes with ferric iron within transferrin and lactoferrin (Sandrini et al., 2010). Subsequently, $\mathrm{Fe}^{+3}$ bound to the transferrin enzyme is reduced to the $\mathrm{Fe}^{+2}$ that accompanies the release of free iron. In the presence of norepinephrine, Holo-transferrin, the iron-replete form of transferrin progressively releases iron and undergoes conversion to the iron-depleted apo-transferrin form. These changes are evident by both electron paramagnetic resonance spectrometry as well as PAGE. Sandrini et al. (2010) reported parallel findings for both transferrin and lactoferrin, and it is thus likely that a reductive mechanism of iron release is common to the liberation of iron from both enzymes. Whereas these findings provide for a mechanism by which stress-related neurochemicals influence the physiology of microorganisms, other noniron-related mechanisms may also be involved. For example, the first description that prokaryotic microbes possess a plasma membrane monoamine transporter and an organic cation transporter capable of facilitating the uptake of neurochemicals such as NE has been recently published (Lyte and Brown, 2018). This finding of a hitherto unrecognized mechanism by which a bacterium may interact with host signals demonstrates that multiple mechanisms exist that microorganisms may use to interact with host neurochemicals [see Lyte (2016b) for a more comprehensive review].

Third, other research groups (Curtis et al., 2014; Moreira et al., 2016) have suggested that NE can interact with a quorum sensor histidine kinase (QseC) that is present in bacteria. The QseC is linked to genes encoding bacterial virulence factors as well as genes whose protein products control aspects of bacterial growth and colony density. Animal pathogens that possess QseC or a QseC homolog include E. coli, Shigella, Salmonella, Klebsiella, Vibrio, Pseudomonas, Acinetobacter, Francisella, and Yersinia. These pathogens have developed a synthetic allosteric modulator of this quorum sensor kinase, which has been shown to inhibit the effects of NE (Rasko and Sperandio, 2010). The QseC does not have the pharmacological characteristics of an adrenergic receptor because it has relatively low affinity for NE, is not inhibited by conventional adrenergic receptor antagonists at low concentrations, and is not particularly stereoselective. At a high concentration of $5 \mathrm{~m} M$, NE increased E. coli O157:H7 adherence as well as intestinal fluid secretion and neutrophil influx when introduced with these bacteria into isolated ileal loops of anesthetized bull calves. This effect was dependent upon the expression of virulence genes in this bacterial strain necessary to form attaching and effacing lesions (Vlisidou et al., 2004). The finding is consistent with the concept that QseC is linked to the expression of virulence genes in enterohemorrhagic $E$. coli.

Fourth, NE acting on bacterial QseC can induce the production of bacterial enzymes that oxidize locally produced NE to its common metabolite, 3,4-dihydroxymandelic acid (DHMA). This substance can act as a potent chemotactic agent in bacterial species expressing the serine chemoreceptor, Tsr (Figure 3). Bacteria such as E. coli that possess Tsr can follow 3,4-dihydroxymandelic acid up its concentration gradient to the bacteria from which it was expressed. Through this mechanism, it has been proposed that bacteria stimulated by $\mathrm{NE}$ to adhere to the intestinal epithelium through a QseCdependent mechanism could then oxidize NE and call other bacteria to join them at the mucosal surface (Pasupuleti et al., 2014). Intestinal regions that appear to favor bacterial colonization include the perifollicular areas of discrete Peyer's patches in the upper small intestine; these areas have a relatively high density of sympathetic nerves (Green et al., 2003; Chiocchetti et al., 2008).

Whereas it is clear that catecholamines have the potential to directly act on bacteria to promote growth and virulence gene expression, the mechanism(s) by which these effects are affected are still incompletely understood. We have already touched on 2 hypotheses, (1) the capacity for catecholamines to facilitate the supply of iron under iron-limiting conditions and (2) the ability for catecholamines to interact with the quorum sensor histidine kinase QsecC. However, it must be emphasized that another mechanism(s) may exist by which catecholamines and catecholamine-based synthetic drugs could interact with microorganisms. 
Norepinephrine enhances the in vivo replication of Salmonella enterica serovar Typhimurium within the bovine ileum (Pullinger et al., 2010). Neither QesC nor QseE are required for this effect and mutant knockouts $\Delta q s e c C, \Delta q s e E$, and $\triangle q s e C E$ all retain a higher rate of in vivo net replication in response to NE. Although an iron uptake-related phenomena may partially explain this finding, it is also conceivable that some other mechanism might account for this growth enhancement effect. The catecholamines dopamine, NE, and epinephrine all have the capacity to chelate iron through the cis-diol portion of the catechol moiety. However, the catechol moiety is also present in the catecholamine precursor and L-DOPA (L-3,4-dihydroxyphenylalanine) has the capacity to complex with iron (Linert et al., 1991). Because many plant-derived foodstuffs contain significant quantities of L-DOPA or the L-DOPA precursor tyrosine (readily converted to L-DOPA by bacterial tyrosine hydrolase), L-DOPA is likely to be available in at least some portions of the ruminant digestive tract. Given this finding, it would seem counterintuitive that there would be a special advantage in utilizing catecholamines, such as NE or dopamine, for iron uptake when a potentially suitable uptake molecule, such as L-DOPA, might be available. Structurally, there is little reason to believe any great differences exist in iron-binding capacity between L-DOPA, dopamine, norepinephrine, and epinephrine. No study has yet examined the pos- sible differences between L-DOPA and its downstream products dopamine, epinephrine, and NE.

Idiosyncratic effects distinct to individual catecholamines have nevertheless been observed. The bacterial pathogen Actinobacillus pleuropneumoniae manifests differential expression of 158 and 105 genes when exposed to epinephrine or NE, respectively (Li et al., 2012). This finding suggests that some bacteria are capable of distinguishing between different catecholamines. As adrenergic sensor kinases such as QseC and QseE cannot account for all of these observations, it seems probable that other recognition and signaling mechanisms exist. Whatever the case may be, NE can be present in intestinal luminal fluids at concentrations that can act on bacteria to enhance their growth, virulence, and interactions with entero-adherent bacteria. The means by which neurochemicals may achieve these changes could be multifactorial, with new, novel mechanisms yet to be elucidated. The results of studies of NE on the intestinal mucosa, especially those performed in swine described above, could be quite applicable to understanding the actions of NE and other catecholamines in the dairy cow intestinal mucosa. Many opportunities remain to investigate the ability of stress and the consequent production of catecholamines to influence cow health. These studies should extend to the role of stress and catecholamines in modifying the susceptibility of dairy cattle to infectious diseases

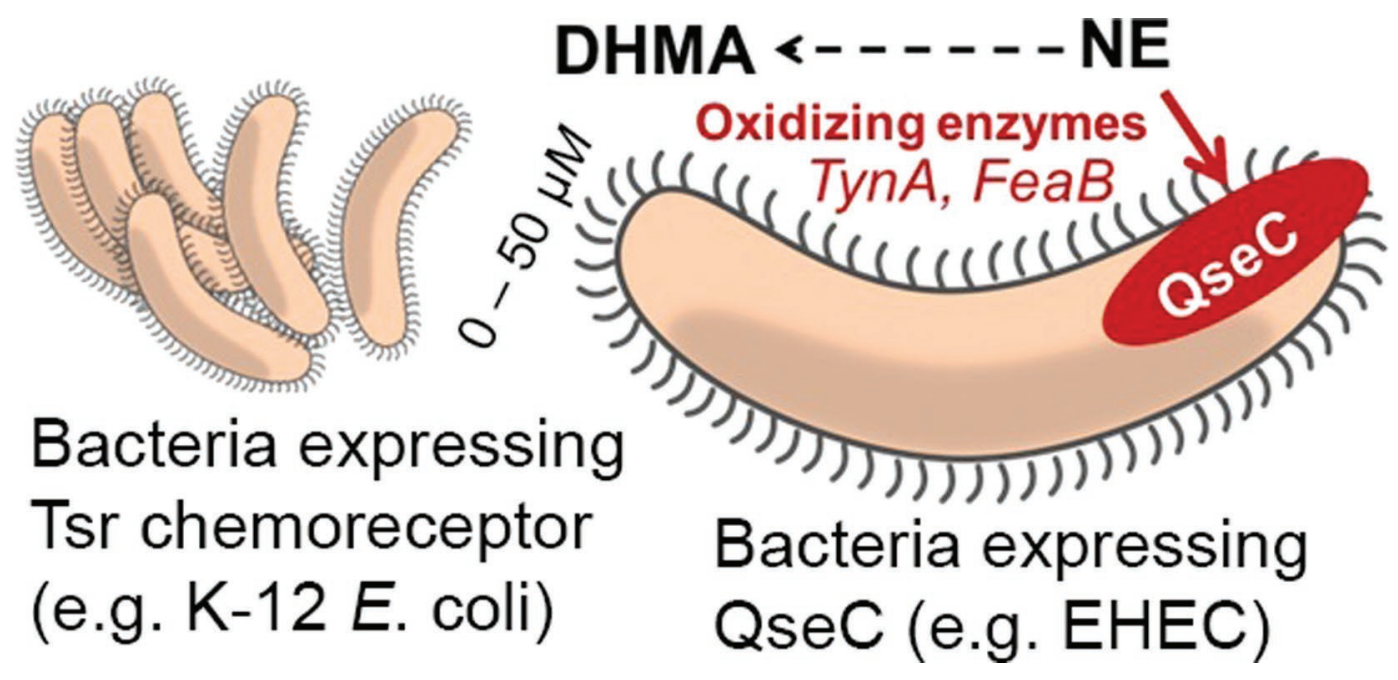

Figure 3. Norepinephrine (NE) can interact with the quorum sensor histidine kinase (QseC) that is expressed in several bacterial species. QseC is linked to genes encoding bacterial virulence factors as well as genes whose protein products control aspects of bacterial growth and colony density. When activated by NE, QseC can induce the production of bacterial enzymes (TynA, FeaB) that catalyze the oxidation of locally produced NE to its common metabolite, 3,4-dihydroxymandelic acid (DHMA). This latter substance can act as a potent chemotactic signal in bacterial species that express the serine chemoreceptor (Tsr). Bacteria such as Escherichia coli that possess Tsr can follow DHMA up its concentration gradient to the bacteria that expressed it. Through this mechanism, it has been proposed that bacteria stimulated by NE to adhere to the intestinal epithelium through a QseC-dependent mechanism could subsequently oxidize NE and call other bacteria to join them at the mucosal surface (Pasupuleti et al., 2014). EHEC = enterohemorrhagic E. coli. Color version available online. 
through their ability to alter the gut microbial community.

\section{DRAWING PARALLELS BETWEEN NONRUMINANTS AND RUMINANTS}

Unlike the alimentary tracts of mammals such as humans or rodents, those of dairy cattle are uniquely complex with 4 highly specialized forestomaches. As such, the microbial biogeography of the bovine alimentary tract displays a commensurate increase in relative complexity. For example, studies have shown more than twice the number of operational taxonomic units in the foregut as in the intestinal structures past the stomach (Mao et al., 2015). Under normal (healthy) conditions, a common mammalian microbiota is dominated by Firmicutes (65.7\% by sequencing), Bacteroidetes (16.3\%), Proteobacteria (8.8\%), and Actinobacteria (4.7\%; Ley et al., 2008). Likewise, in cattle, Firmicutes, Bacteroidetes, and Proteobacteria predominate (Bickhart and Weimer, 2018). However, the composition, population dynamics, and functions of microbial communities can vary radically between different gut locations and are affected by diet and by physiological and health status of the cow (Bickhart and Weimer, 2018; Pitta et al., 2018). The relative abundances of genes involved in carbohydrate metabolism are heavily represented in digesta samples from the forestomaches, whereas genes related to AA metabolism are enriched in mucosal samples (Mao et al., 2015). Given observations of this nature and differences in specific functions of the digestive tract (Steele et al., 2016), it is reasonable to infer that the unique microbial behavior and diversity inherent to these regions have the capacity to influence feed efficiency and other metabolic characteristics (Bravo and Wall, 2016). It is also likely that interactions between the cow and its rumen microbes could be manipulated to improve cattle productivity and well-being (Bickhart and Weimer, 2018).

Though the structures of the forestomaches may lack the dense innervation of more distal gastrointestinal structures, this does not mean resident organisms that produce neurochemicals or other signaling molecules in these compartments cannot widely influence host status. The rumen lining consists of keratinized stratified tissue, unlike the simple columnar epithelium associated with most intestinal sites. Though the absorptive features of the rumen are distinct from gastrointestinal regions lined with mucosal surfaces, the transport of many molecules from the rumen, including short-chain fatty acids such as acetate, propionate, and butyrate generated by intraruminal microbial fermentation, has been well established (Graham and Simmons, 2005; Baldwin and Connor, 2017).
Electrolyte transport of $\mathrm{Na}^{+}$via the distribution of the $\mathrm{Na}^{+}-\mathrm{K}^{+}$-ATPase is also well documented. Though we know of no studies that specifically document the ability of catecholamines to influence these processes in the rumen, we have already noted that catecholamines have the potential to effect changes in the growth and metabolism of various microbes. As a corollary, it follows that effects on elements of the microbiota responsible for producing short-chain fatty acids could dramatically alter animal productivity. Furthermore, as described above, NE and dopamine can influence mucosal ion and water transport throughout the digestive tract (Zhang et al., 2007). If similar findings can be documented in the rumen, then it may well follow that the microbiota has the potential to affect other absorptive pathways through osmotic and gradient driven influences. Though such mechanisms can be imagined through the lens of microbial endocrinology, research is needed to substantiate any specific hypotheses.

Exposure of animals to stressful stimuli has been found to produce changes in the gut microbiota (Mackos et al., 2017). The release of catecholamines from injured enteric neurons is associated with a rapid alteration of the microbiota community from one dominated by gram-positive taxa to one dominated by a single gram-negative bacterial species, Escherichia coli (Lyte and Bailey, 1997). In some species, decreased microbial diversity has been associated with health problems ranging from recurrent Clostridium difficile infection to metabolic disturbances (Chang et al., 2008; Claesson et al., 2012). Given a possible link between microbial diversity and the overall metabolic state of the host, it is not a wide leap to suggest signals that alter this balance may also have the potential to more profoundly affect the metabolism of the host.

Clostridium perfringens, the agent responsible for the majority of cases of enterotoxemia in cattle as well as the highly fatal jejunal hemorrhage syndrome (Elhanafy et al., 2013) holds an interesting parallel with some hallmark observations in the field of microbial endocrinology. In most cases of this syndrome, $C$. perfringens can be isolated from lesions and intestinal contents; however, simply inoculating the purified organism into an animal does not reproduce the disease (Abutarbush and Radostits, 2005). Interestingly, C. perfringens is also the earliest reported example of a microorganism whose growth is influenced by a neurochemical. During the early 20th century, the drainage of bacterial abscesses and administration of drugs was often achieved through the usage of reusable glass syringes. Cleaning methods of the day left trace quantities of $C$. perfringens, but this typically was inconsequential, as the spores were mostly dormant. In the early 1930 s, the catecholamine epinephrine was the first manufactured 
hormone to be used in the clinical setting. Soon after this development, reports began to appear of patients suffering from fulminating sepsis hours after treatment with epinephrine. Epinephrine increases the growth rate of $C$. perfringens and decreases the infective dose by up to 1 million fold (Renaud, 1930; Cooper, 1946). Utilizing a perspective based in microbial endocrinology, it can be suggested that successful infection may be dependent not just on the presence of the infectious agent, but also the presence of a signaling host factor, such as a stress-derived catecholamine, that could initiate bacterial virulence. In fact, expression of the K99 pilus adhesin of enterotoxigenic $E$. coli was shown to be increased over 280 fold in the presence of $\mathrm{NE}$, thereby greatly enhancing its ability to attach to the intestinal mucosa and initiate infection (Lyte et al., 1997).

\section{FUTURE AVENUES OF STUDY AND AN APPLICATION OF THE PRINCIPLES OF MICROBIAL ENDOCRINOLOGY TO RUMINANT HEALTH}

Presently, the application of microbial endocrinologybased concepts to the alimentary tract of dairy cattle is still relatively new, with experiments examining the ruminal environment in the formative stage. Little is known concerning the neurohormonal innervation of the alimentary tract, although the ruminal epithelium does not possess the neurohormonal signaling circuitry that is present in the intestinal mucosa (Kitamura et al., 1987; Kitamura et al., 1993; Teixeira et al., 1998). There are, however, a few examples of research involving neuroendocrine-based applications such as the use of beta-adrenergic agonists to improve gain and efficiency in ruminants during the final days prior to harvest (Walker and Drouillard, 2012). As such, we do not know how microbial endocrinology concepts might apply to the ruminal microbial environment. However, interactions between epimural microorganisms and chemical signals released from discrete strata of ruminal epithelial cells may exist. Although our short review has mainly been concerned with stress-derived neurochemicals, principally NE, other microbially derived hormones and signaling molecules have been demonstrated. For example, lactobacilli and bifidobacteria have been shown to produce $\gamma$-aminobutyric acid; Candida, Streptococcus, Escherichia, and Enterococcus can synthesize serotonin; and lactobacilli, lactococci, streptococci, and enterococci are capable of making histamine (for a complete description of the range of microbially produced neurochemicals see Lyte, 2016a). Given the enormous microbial diversity of the ruminal compartments, it is possible that ruminant microbes harbor many of the synthetic pathways requisite for these chemicals. The physiological significance of such production in ruminants is as yet unexplored. Similarly, the capacity for some groups of bacteria to produce biologically active molecules such as neurochemicals has the capacity to shape broader microbial communities, which remains a possible avenue for even relatively small groups of bacteria might influence a host as large as a ruminant.

One avenue of potential research suggested by the application of a microbial endocrinology-based approach to ruminant health is the mining of the genomic and metabolomic databases that have been established. For example, bovine metabolome databases have recently been established (Goldansaz et al., 2017; www .cowmetdb.ca and www.lmdb.ca). The use of such databases, however, should be approached with caution due to the well-recognized challenges that are inherent in the use of such databases (Smirnov et al., 2016).

As studies specifically testing microbial endocrinology-based interventions in ruminants are currently few, it seems premature to offer any specific advice in regard to animal husbandry practices beyond the evident need to limit stress, as stressful conditions could expose the ruminant digestive tract to stress-related catecholamines such as NE at high concentrations. The realization that members of gut microbial communities have the capacity to generate neurochemicals from foodstuffs does suggest that a closer examination of feed content may lead to dietary modifications.

Skilled cattle farmers are aware of the importance of the microbiome-diet connection in conditions such as ruminal acidosis. In this condition, an overabundance of simple sugars rapidly changes the microbial community structure, which in turn generates copious amounts of fermented products and acids that can lead to serious health problems. Perhaps a closer inspection of feed from the neurochemical perspective will shine a light on other conditions that could be related to an overabundance of neurochemicals and their precursors. For example, consider that the neurochemical precursor tyrosine is a common component of most animal feed sources including grains. Farmers wishing to avoid some of the complications of stress in their animals might select less tyrosine-rich foods if they learn that tyrosine could heighten aggression in stressed individuals (Lieberman et al., 2015). Likewise, improved understanding of alterations in resident microbiota during growth (Meale et al., 2017), during metabolic disorders such as milk fat depression (Pitta et al., 2018), and during stressful conditions (Kvidera et al., 2017) will likely increase the number of studies that assess host-microbiome interactions. Although in its infancy, great potential exists for the examination of microbial endocrinology-based mechanisms within the alimentary 
tract to yield valuable new information that could translate into new methods and management strategies to improve the well-being and productivity of dairy cattle and other ruminant species.

\section{REFERENCES}

Abutarbush, S. M., and O. M. Radostits. 2005. Jejunal hemorrhage syndrome in dairy and beef cattle: 11 cases (2001 to 2003). Can. Vet. J. 46:711-715.

Asano, Y., T. Hiramoto, R. Nishino, Y. Aiba, T. Kimura, K. Yoshihara, Y. Koga, and N. Sudo. 2012. Critical role of gut microbiota in the production of biologically active, free catecholamines in the gut lumen of mice. Am. J. Physiol. Gastrointest. Liver Physiol. 303:G1288-G1295.

Atuma, C., V. Strugala, A. Allen, and L. Holm. 2001. The adherent gastrointestinal mucus gel layer: thickness and physical state in vivo. Am. J. Physiol. Gastrointest. Liver Physiol. 280:G922-G929.

Baldwin, R. L. t., and E. E. Connor. 2017. Rumen function and development. Vet. Clin. North Am. Food Anim. Pract. 33:427-439.

Baumgard, L. H., and R. P. Rhoads. 2012. Ruminant nutrition symposium: ruminant production and metabolic responses to heat stress. J. Anim. Sci. 90:1855-1865.

Baumgard, L. H., and R. P. Rhoads Jr.. 2013. Effects of heat stress on postabsorptive metabolism and energetics. Annu. Rev. Anim. Biosci. 1:311-337.

Bernabucci, U., N. Lacetera, L. H. Baumgard, R. P. Rhoads, B. Ronchi, and A. Nardone. 2010. Metabolic and hormonal acclimation to heat stress in domesticated ruminants. Animal 4:1167-1183.

Bickhart, D. M., and P. J. Weimer. 2018. Host-rumen microbe interactions may be leveraged to improve the productivity of dairy cows. J. Dairy Sci. 101:1-10. https://doi.org/10.3168/jds.2017-13328.

Bradford, B. J., K. Yuan, J. K. Farney, L. K. Mamedova, and A. J. Carpenter. 2015. Invited review: Inflammation during the transition to lactation: New adventures with an old flame. J. Dairy Sci. 98:6631-6650.

Bradford, B. J., K. Yuan, and C. Ylioja. 2016. Managing complexity: Dealing with systemic crosstalk bovine physiology. J. Dairy Sci. 99:4983-4996.

Bravo, D. M., and E. H. Wall. 2016. The rumen and beyond: Nutritional physiology of the modern dairy cow. J. Dairy Sci. 99:49394940.

Brown, D. R., and S. M. O'Grady. 1997. Regulation of ion transport in the porcine intestinal tract by enteric neurotransmitters and hormones. Comp. Biochem. Physiol. A Physiol. 118:309-317.

Chang, J. Y., D. A. Antonopoulos, A. Kalra, A. Tonelli, W. T. Khalife, T. M. Schmidt, and V. B. Young. 2008. Decreased diversity of the fecal microbiome in recurrent Clostridium difficile-associated diarrhea. J. Infect. Dis. 197:435-438.

Chen, C., M. Lyte, M. P. Stevens, L. Vulchanova, and D. R. Brown. 2006. Mucosally-directed adrenergic nerves and sympathomimetic drugs enhance non-intimate adherence of Escherichia coli O157:H7 to porcine cecum and colon. Eur. J. Pharmacol. 539:116-124.

Chiocchetti, R., G. Mazzuoli, V. Albanese, M. Mazzoni, P. Clavenzani, G. Lalatta-Costerbosa, M. L. Lucchi, G. Di Guardo, G. Marruchella, and J. B. Furness. 2008. Anatomical evidence for ileal Peyer's patches innervation by enteric nervous system: A potential route for prion neuroinvasion? Cell Tissue Res. 332:185-194.

Claesson, M. J., I. B. Jeffery, S. Conde, S. E. Power, E. M. O'Connor, S. Cusack, H. M. Harris, M. Coakley, B. Lakshminarayanan, O. O'Sullivan, G. F. Fitzgerald, J. Deane, M. O'Connor, N. Harnedy, K. O'Connor, D. O'Mahony, D. van Sinderen, M. Wallace, L. Brennan, C. Stanton, J. R. Marchesi, A. P. Fitzgerald, F. Shanahan, C. Hill, R. P. Ross, and P. W. O'Toole. 2012. Gut microbiota composition correlates with diet and health in the elderly. Nature 488:178-184

Clarke, M. B., D. T. Hughes, C. Zhu, E. C. Boedeker, and V. Sperandio. 2006. The QseC sensor kinase: A bacterial adrenergic receptor. Proc. Natl. Acad. Sci. USA 103:10420-10425.
Collier, R. J., G. E. Dahl, and M. J. VanBaale. 2006. Major advances associated with environmental effects on dairy cattle. J. Dairy Sci. 89:1244-1253.

Cooper, E. V. 1946. Gas-gangrene following injection of adrenaline. Lancet 1:459-461.

Curtis, M. M., R. Russell, C. G. Moreira, A. M. Adebesin, C. Wang, N. S. Williams, R. Taussig, D. Stewart, P. Zimmern, B. Lu, R. N. Prasad, C. Zhu, D. A. Rasko, J. F. Huntley, J. R. Falck, and V. Sperandio. 2014. QseC inhibitors as an antivirulence approach for gram-negative pathogens. MBio 5:e02165.

Elhanafy, M. M., D. D. French, and U. Braun. 2013. Understanding jejunal hemorrhage syndrome. J. Am. Vet. Med. Assoc. 243:352-358.

Ellermann, M., and J. C. Arthur. 2017. Siderophore-mediated iron acquisition and modulation of host-bacterial interactions. Free Radic. Biol. Med. 105:68-78.

Freestone, P. P., R. A. Hirst, S. M. Sandrini, F. Sharaff, H. Fry, S. Hyman, and C. O'Callaghan. 2012. Pseudomonas aeruginosacatecholamine inotrope interactions: A contributory factor in the development of ventilator-associated pneumonia? Chest 142:12001210.

Furness, J. B. 2016. Integrated neural and endocrine control of gastrointestinal function. Adv. Exp. Med. Biol. 891:159-173.

Furness, J. B., L. R. Rivera, H. J. Cho, D. M. Bravo, and B. Callaghan. 2013. The gut as a sensory organ. Nat. Rev. Gastroenterol. Hepatol. 10:729-740.

Goldansaz, S. A., A. C. Guo, T. Sajed, M. A. Steele, G. S. Plastow, and D. S. Wishart. 2017. Livestock metabolomics and the livestock metabolome: A systematic review. PLoS One 12:e0177675.

Graham, C., and N. L. Simmons. 2005. Functional organization of the bovine rumen epithelium. Am. J. Physiol. Regul. Integr. Comp. Physiol. 288:R173-R181.

Green, B. T., M. Lyte, C. Chen, Y. Xie, M. A. Casey, A. KulkarniNarla, L. Vulchanova, and D. R. Brown. 2004. Adrenergic modulation of Escherichia coli O157:H7 adherence to the colonic mucosa. Am. J. Physiol. Gastrointest. Liver Physiol. 287:G1238-G1246.

Green, B. T., M. Lyte, A. Kulkarni-Narla, and D. R. Brown. 2003. Neuromodulation of enteropathogen internalization in Peyer's patches from porcine jejunum. J. Neuroimmunol. 141:74-82.

Hughes, D. T., and V. Sperandio. 2008. Inter-kingdom signaling: Communication between bacteria and their hosts. Nat. Rev. Microbiol. 6:111-120.

Jing, L., R. Zhang, Y. Liu, W. Zhu, and S. Mao. 2014. Intravenous lipopolysaccharide challenge alters ruminal bacterial microbiota and disrupts ruminal metabolism in dairy cattle. Br. J. Nutr. 112:170-182

Kitamura, N., J. Yamada, Y. Yamamoto, and T. Yamashita. 1993. Substance P-immunoreactive neurons the bovine forestomach mucosa: their presumptive role in a sensory mechanism. Arch. Histol. Cytol. 56:399-410.

Kitamura, N., J. Yamada, and T. Yamashita. 1987. Immunohistochemical study on the distribution of neuron-specific enolase- and peptide-containing nerves in the omasum of cattle. J. Comp. Neurol. 256:590-599.

Kvidera, S. K., M. J. Dickson, M. Abuajamieh, D. B. Snider, M. V. S. Fernandez, J. S. Johnson, A. F. Keating, P. J. Gorden, H. B. Green, K. M. Schoenberg, and L. H. Baumgard. 2017. Intentionally induced intestinal barrier dysfunction causes inflammation, affects metabolism, and reduces productivity in lactating Holstein cows. J. Dairy Sci. 100:4113-4127.

Ley, R. E., M. Hamady, C. Lozupone, P. Turnbaugh, R. R. Ramey, J. S. Bircher, M. L. Schlegel, T. A. Tucker, M. D. Schrenzel, R. Knight, and J. I. Gordon. 2008. Evolution of mammals and their gut microbes. Science 320:1647-1651.

Li, L., Z. Xu, Y. Zhou, L. Sun, Z. Liu, H. Chen, and R. Zhou. 2012. Global effects of catecholamines on Actinobacillus pleuropneumoniae gene expression. PLoS One 7:e31121.

Lieberman, H. R., L. A. Thompson, C. M. Caruso, P. J. Niro, C. R. Mahoney, J. P. McClung, and G. R. Caron. 2015. The catecholamine neurotransmitter precursor tyrosine increases anger during exposure to severe psychological stress. Psychopharmacology (Berl.) 232:943-951. 
Linert, W., R. F. Jameson, and E. Herlinger. 1991. Complex formation followed by internal electron transfer: the reaction between L-dopa and iron(III). Inorg. Chim. Acta 187:239-247.

Lyte, M. 2014. Microbial endocrinology and the microbiota-gut-brain axis. Adv. Exp. Med. Biol. 817:3-24.

Lyte, M. 2016a. Microbial endocrinology. Pages $89-108$ in The GutBrain Axis. C. Stanton, ed. Academic Press, Cambridge, MA.

Lyte, M. 2016b. Microbial endocrinology in the pathogenesis of infectious disease. Microbiol. Spectr. 4:1-2.

Lyte, M., and M. T. Bailey. 1997. Neuroendocrine-bacterial interactions in a neurotoxin-induced model of trauma. J. Surg. Res. 70:195-201.

Lyte, M., and D. R. Brown. 2018. Evidence for PMAT- and OCT-like biogenic amine transporters in a probiotic strain of Lactobacillus: Implications for interkingdom communication within the microbiota-gut-brain axis. PLoS One 13:e191037.

Lyte, M., A. K. Erickson, B. P. Arulanandam, C. D. Frank, M. A. Crawford, and D. H. Francis. 1997. Norepinephrine-induced expression of the K99 pilus adhesin of enterotoxigenic Escherichia coli. Biochem. Biophys. Res. Commun. 232:682-686.

Lyte, M., and S. Ernst. 1992. Catecholamine induced growth of gram negative bacteria. Life Sci. 50:203-212.

Mackos, A. R., R. Maltz, and M. T. Bailey. 2017. The role of the commensal microbiota in adaptive and maladaptive stressor-induced immunomodulation. Horm. Behav. 88:70-78.

Malmuthuge, N., and L. L. Guan. 2017. Understanding host-microbial interactions in rumen: searching the best opportunity for microbiota manipulation. J. Anim. Sci. Biotechnol. 8:8.

Mao, S., M. Zhang, J. Liu, and W. Zhu. 2015. Characterizing the bacterial microbiota across the gastrointestinal tracts of dairy cattle: membership and potential function. Sci. Rep. 5:16116.

McCowan, R. P., K. J. Cheng, C. B. Bailey, and J. W. Costerton. 1978. Adhesion of bacteria to epithelial cell surfaces within the reticulo-rumen of cattle. Appl. Environ. Microbiol. 35:149-155.

Meale, S. J., F. Chaucheyras-Durand, H. Berends, L. L. Guan, and M. A. Steele. 2017. From pre- to postweaning: Transformation of the young calf's gastrointestinal tract. J. Dairy Sci. 100:5984-5995.

Moreira, C. G., R. Russell, A. A. Mishra, S. Narayanan, J. M. Ritchie, M. K. Waldor, M. M. Curtis, S. E. Winter, D. Weinshenker, and V. Sperandio. 2016. Bacterial adrenergic sensors regulate virulence of enteric pathogens in the gut. MBio 7:e00826.

Mueller, R. E., J. M. Asplund, and E. L. Iannotti. 1984. Successive changes in the epimural bacterial community of young lambs as revealed by scanning electron microscopy. Appl. Environ. Microbiol. 47:715-723.

Pasupuleti, S., N. Sule, W. B. Cohn, D. S. MacKenzie, A. Jayaraman, and M. D. Manson. 2014. Chemotaxis of Escherichia coli to norepinephrine (NE) requires conversion of NE to 3,4-dihydroxymandelic acid. J. Bacteriol. 196:3992-4000.

Pessione, E., and S. Cirrincione. 2016. Bioactive molecules released in food by lactic acid bacteria. Encrypted peptides and biogenic amines. Front. Microbiol. 7:876.

Pitta, D. W., N. Indugu, B. Vecchiarelli, D. E. Rico, and K. J. Harvatine. 2018. Alterations in ruminal bacterial populations at induction and recovery from diet-induced milk fat depression in dairy cows. J. Dairy Sci. 101:295-309.

Pullinger, G. D., S. C. Carnell, F. F. Sharaff, P. M. van Diemen, F. Dziva, E. Morgan, M. Lyte, P. P. Freestone, and M. P. Stevens 2010. Norepinephrine augments Salmonella enterica-induced enteritis in a manner associated with increased net replication but independent of the putative adrenergic sensor kinases QseC and QseE. Infect. Immun. 78:372-380.
Rasko, D. A., and V. Sperandio. 2010. Anti-virulence strategies to combat bacteria-mediated disease. Nat. Rev. Drug Discov. 9:117128.

Renaud, M. M. A. 1930. Role favorisant des perturbations locales causes par l' adrenaline sur led developpement des infections microbiennes. C. R. Seances Soc. Biol. Fil. 103:1052-1054.

Sandrini, S. M., R. Shergill, J. Woodward, R. Muralikuttan, R. D. Haigh, M. Lyte, and P. P. Freestone. 2010. Elucidation of the mechanism by which catecholamine stress hormones liberate iron from the innate immune defense proteins transferrin and lactoferrin. J. Bacteriol. 192:587-594.

Schmidt, L. D., Y. Xie, M. Lyte, L. Vulchanova, and D. R. Brown. 2007. Autonomic neurotransmitters modulate immunoglobulin A secretion in porcine colonic mucosa. J. Neuroimmunol. 185:20-28.

Schmidt, P. T., L. Eriksen, M. Loftager, T. N. Rasmussen, and J. J Holst. 1999. Fast acting nervous regulation of immunoglobulin A secretion from isolated perfused porcine ileum. Gut 45:679-685.

Schroeder, B. O., and F. Backhed. 2016. Signals from the gut microbiota to distant organs in physiology and disease. Nat. Med. 22:1079-1089.

Sharon, G., N. Garg, J. Debelius, R. Knight, P. C. Dorrestein, and S. K. Mazmanian. 2014. Specialized metabolites from the microbiome in health and disease. Cell Metab. 20:719-730.

Silla Santos, M. H. 1996. Biogenic amines: Their importance in foods. Int. J. Food Microbiol. 29:213-231.

Smirnov, K. S., T. V. Maier, A. Walker, S. S. Heinzmann, S. Forcisi, I. Martinez, J. Walter, and P. Schmitt-Kopplin. 2016. Challenges of metabolomics in human gut microbiota research. Int. J. Med. Microbiol. 306:266-279.

Steele, M. A., G. B. Penner, F. Chaucheyras-Durand, and L. Guan. 2016. Development and physiology of the rumen and the lower gut: Targets for improving gut health. J. Dairy Sci. 99:4955-4966.

Sutherland, D. B., K. Suzuki, and S. Fagarasan. 2016. Fostering of advanced mutualism with gut microbiota by immunoglobulin A. Immunol. Rev. 270:20-31

Teixeira, A. F. T. Wedel, H. J. Krammer, and W. Kuhnel. 1998. Structural differences of the enteric nervous system in the cattle forestomach revealed by whole mount immunohistochemistry. Ann. Anat. 180:393-400.

Uyeno, Y., Y. Sekiguchi, K. Tajima, A. Takenaka, M. Kurihara, and Y. Kamagata. 2010. An rRNA-based analysis for evaluating the effect of heat stress on the rumen microbial composition of Holstein heifers. Anaerobe 16:27-33.

Vlisidou, I., M. Lyte, P. M. van Diemen, P. Hawes, P. Monaghan, T. S. Wallis, and M. P. Stevens. 2004. The neuroendocrine stress hormone norepinephrine augments Escherichia coli O157:H7-induced enteritis and adherence in a bovine ligated ileal loop model of infection. Infect. Immun. 72:5446-5451.

Walker, C. E., and J. Drouillard. 2012. Effects of catecholamines on gut microflora and potential for beta- adrenergic agonists to impact ruminal fermentation. Open Agric. J. 6:57-66.

Wetzels, S. U., E. Mann, B. U. Metzler-Zebeli, P. Pourazad, M. Qumar, F. Klevenhusen, B. Pinior, M. Wagner, Q. Zebeli, and S. SchmitzEsser. 2016. Epimural indicator phylotypes of transiently-induced subacute ruminal acidosis in dairy cattle. Front. Microbiol. 7:274.

Zhang, G. H., J. X. Zhu, H. Xue, J. Fan, X. Chen, L. L. Tsang, Y W. Chung, Y. Xing, and H. C. Chan. 2007. Dopamine stimulates $\mathrm{Cl}(-)$ absorption coupled with $\mathrm{HCO}(3)(-)$ secretion in rat late distal colon. Eur. J. Pharmacol. 570:188-195. 